\title{
Carriers of nickel nucleosynthetic anomalies uncovered by a step leaching experiment
}

\author{
GEORGY V. MAKHATADZE, MARTIN SCHILLER AND \\ MARTIN BIZZARRO
}

Centre for Star and Planet Formation, Globe Institute, University of Copenhagen

Presenting Author: georgy.makhatadze@sund.ku.dk

Nucleosynthetic isotope heterogeneity in Solar System solids, asteroids and planetary bodies can be explained by either unmixing or imperfect mixing of presolar carriers produced by distinct stellar sources. A better understanding of the nature of the carrier phases as well as the origin of the nucleosynthetic heterogeneity can be obtained through the study of primitive meteorites. In particular, CI chondrites are pristine, thermally unprocessed meteorites believed to represent the bulk Solar System composition and, as such, are ideal target for search for carriers of isotope anomalies. Here, we present high-precision mass-independent $\mathrm{Ni}$ isotope data of step-leaches of the Ivuna CI chondrite previously analyzed for a number of nuclides [1]. Additionally, we present data for bulk chondrites and CAI. Our data were obtained by high-resolution MC-ICPMS using the ${ }^{62} \mathrm{Ni} /{ }^{61} \mathrm{Ni}$ for normalization since it has been shown that ${ }^{58} \mathrm{Ni}$ exhibits the widest range of anomalies [2].

Five of the 11 leaches define highly variable $\mu^{58} \mathrm{Ni}, \mu^{60} \mathrm{Ni}$ and $\mu^{64} \mathrm{Ni}$ compositions that allow to identify four distinct nucleosynthetic components. In detail, L6 records a deficit of $\sim 80 \mathrm{ppm}$ in $\mu^{64} \mathrm{Ni}$ whereas L8-L9 show excesses in $\mu^{64} \mathrm{Ni}$ of $>60$ $\mathrm{ppm}$. The L10 fraction has a negative anomaly on $\mu^{60} \mathrm{Ni}$ of $\sim 130$ ppm and L11 has an excess in $\mu^{64} \mathrm{Ni}(>260 \mathrm{ppm})$ with large deficits in $\mu^{60} \mathrm{Ni}$ and $\mu^{58} \mathrm{Ni}(>1000 \mathrm{ppm})$. While the L11 composition appears dominated by an AGB signature, the remaining three components (L6, L8-L9 and L10) likely reflect various types of supernova sources, including core-collapse and type Ia supernovae. The nucleosynthetic component defined by L11 is likely the endmember responsible for the correlated ${ }^{58} \mathrm{Ni}-{ }^{64} \mathrm{Ni}$ heterogeneity in bulk meteorites. Moreover, the large $\mu^{60} \mathrm{Ni}$ deficit in $\mathrm{L} 10$ implies that the $\mu^{60} \mathrm{Ni}$ variability is nucleosynthetic and unrelated to the decay of ${ }^{60} \mathrm{Fe}$. Unlike leaches, CAIs record $\mu^{58} \mathrm{Ni}, \mu^{60} \mathrm{Ni}$ and $\mu^{64} \mathrm{Ni}$ variability similar to that observed for bulk chondrites. Collectively, our data support a model where thermal processing-based unmixing was one of the major processes responsible for the Solar System's Ni nucleosynthetic heterogeneity.

[1] Schiller, Bizzarro \& Siebert (2020) Science Advances 6, 18.

[2] Steele, Coath, Regelous, Russell \& Elliott (2012) The Astrophysical Journal 758, 59. 